\title{
Qualification of a numerical anthropomorphic model dedicated to radiological accidents
}

\author{
A. ROUX*, E. GAILLARD-LECANU*, J. F. BOTTOLLIER-DEPOIS*, \\ Q. CHAU*, F. TROMPIER*, L. LEBEDEV**
}

(Manuscript received 18 April 2000, revised 9 July 2000, accepted 5 September 2000)

\begin{abstract}
In the case of accidental exposure to ionising radiation, estimation of the absorbed dose in the organism is an important indicator for evaluating the biological consequences. To complete the clinical and biological investigations, physical dosimetry methods can be used to provide an estimate of the absorbed dose and its distribution in the organism. This article describes the development of calculation tools in physical dosimetry, in particular a numerical anthropomorphic phantom which can be adapted to each individual. A standard model was developed from the data available in the ICPR Publication 23. A set of tests was carried out with the model dimensions being modified to study the effect of morphology on the dose calculation. A comparison was made with another standard model used in internal dosimetry. These studies highlight the parameters to be taken into account in the model, depending on whether the accident is local or global.
\end{abstract}

RÉSUMÉ Caractérisation d'un modèle anthropomorphe numérique pour la reconstitution d'accident radiologique.

En cas de surexposition d'un individu aux rayonnements ionisants, la dose absorbée dans l'organisme est un indicateur important pour évaluer les conséquences biologiques. En complément des données cliniques et biologiques, des techniques de dosimétrie physique permettent de fournir une estimation de la dose reçue et de sa distribution dans l'organisme. Cet article décrit le développement d'outils de calcul de dosimétrie physique, en particulier un fantôme anthropomorphe numérique, adaptable à chaque individu. Un modèle standard a été développé à partir des données de la CIPR 23. Une série de tests a été effectuée en faisant varier les dimensions du modèle afin d'étudier l'effet de la morphologie sur le calcul de dose. Une comparaison avec un autre fantôme utilisé pour les calculs en dosimétrie interne a été réalisée. Ces études ont permis de mettre en avant les paramètres à considérer lors d'une modélisation en fonction du type d'accident : global ou localisé.

\section{Introduction}

The analysis of radiological accidents which have occurred during recent decades has revealed that each has its own special features and originality (IAEA, 1998).

* Institul de protection et de súreté nucléaire, DPHD/SD, BP 6, 92265 Fontenay-aux-Roses Ccdex, France.

** Radiation Physics Department, Moscow Physical Engineering Institute, Moscow, Russia. 
This has resulted in the development of a set of techniques and recommendations to be applied by the various specialists concerned when an accident occurs. Most of these accidents are due to radioactive sources and generally involve a small number of people. Between 1944 and 1993, there were 300 accidents resulting in partial or total exposure of individuals requiring medical treatment (Lefaix et Daburon, 1998). Since 1990, there have been over 30 deaths due to accidents involving radioactive sources used for industrial or medical purposes. The most frequently used sources are ${ }^{192} \mathrm{Ir},{ }^{137} \mathrm{Cs}$ and ${ }^{60} \mathrm{Co}$ whose energies range from a few hundred $\mathrm{keV}$ to $1.5 \mathrm{MeV}$ (de Oliveira, 1987). The radiological consequences of accidents involving these various sources can vary widely, since a large number of parameters, such as energy, distance and exposure duration, come into play.

Physical dosimetry and biological dosimetry, which are often complementary, are just two of the many techniques used to estimate doses after a radiological accident, thereby providing valuable information for physicians. The aim of physical dosimetry is to determine dose distributions within the organism and doses to organs. For this to be possible, the accident situation has to be recreated. Several approaches can be used to physically recreate the accident situation:

- experiments, using a model made of "tissue equivalent" material and fitted with dosimeters adapted to the type of radiation, a source and an environment which are as realistic as possible;

- calculations, using either analytical codes or probabilistic computer codes based on the Monte Carlo method.

The method used depends on the type of accident. However, in most cases, reconstruction by computer is more flexible. Computer tools already exist in the medical field, for treatment by radiotherapy for example, but generally speaking, doses can only be calculated for specific configurations. Now, a way has to be found to model various sources (dimensions, shape and energy spectrum) and the morphology and posture of irradiation victims.

In concrete terms, a Computer-Aided Design (CAD) application is used to model the entire geometry of the accident and a Monte Carlo code is used to simulate particle transport.

The main aim of this work was to qualify the numerical anthropomorphic model. To do this, a preliminary study was carried out to verify the data associated with the existing model and make the necessary adjustments to obtain a representation as realistic as possible of reference man (ICRP, 1975). The influence of geometric accuracy, associated with the CAD tool, on the result of the dose calculation was then studied. Then, a series of six models, based on the existing model and of different heights and weights, was created so that the influence of these two 
parameters on the dose calculation result could be studied. Finally, a third study provided the opportunity to compare our anthropomorphic model to other existing models. The computer code was validated during a preliminary study (Roux et al., 2000).

\section{The tools}

Given the constraints imposed by the approach adopted, it was essential to have a powerful CAD tool for constructing a complex anthropomorphic model and the main elements of its environment (source, wall, screen etc.). Furthermore, as far as operational requirements were concerned, the tool had to be interactive and capable of allowing the height and position of the phantom to be changed rapidly to adapt to the particular circumstances of a given accident. Finally, the geometric standard used by the tool had to be compatible with the dose calculation code. A compromise was reached by using the MGED (Multi-device Graphics EDitor) CAD application (BRL, 1992) in conjunction with the MORSE (Multigroup Oak Ridge Stochastic Experiment) Monte Carlo code (Emmett, 1985).

\subsection{CAD: geometric construction}

MGED is a CAD tool based on the principle of combinational geometry which can be used to combine basic solids with simple geometric shapes (spheres, ovoids, parallelepipeds etc.) or more complex ones (for example truncated cones) using Boolean operators. These combinations determine the regions to which specific materials qualified by composition and density will be allocated. Thus, each region becomes a physical element of the overall model, for example an organ of the model, the radiation source, etc. All the regions can then be gathered into groups according to different criteria: materials, part of the body, etc. The functional groups can easily be manipulated, for example they can be rotated, displaced or have scale factors applied to them, so that the geometry can be rapidly adapted to that of the accident.

An anthropomorphic phantom representing a man was created in this way. It consisted of 173 basic solids forming 123 regions (Figs. la and 1b) and including the skeleton and the organs.

\subsection{The MORSE code}

Of all the existing Monte Carlo codes, the MORSE code is the only one with which the standard geometry mentioned above can be used. It is a multi-group 3D transport code for neutrons and photons. Each particle generated by the code 

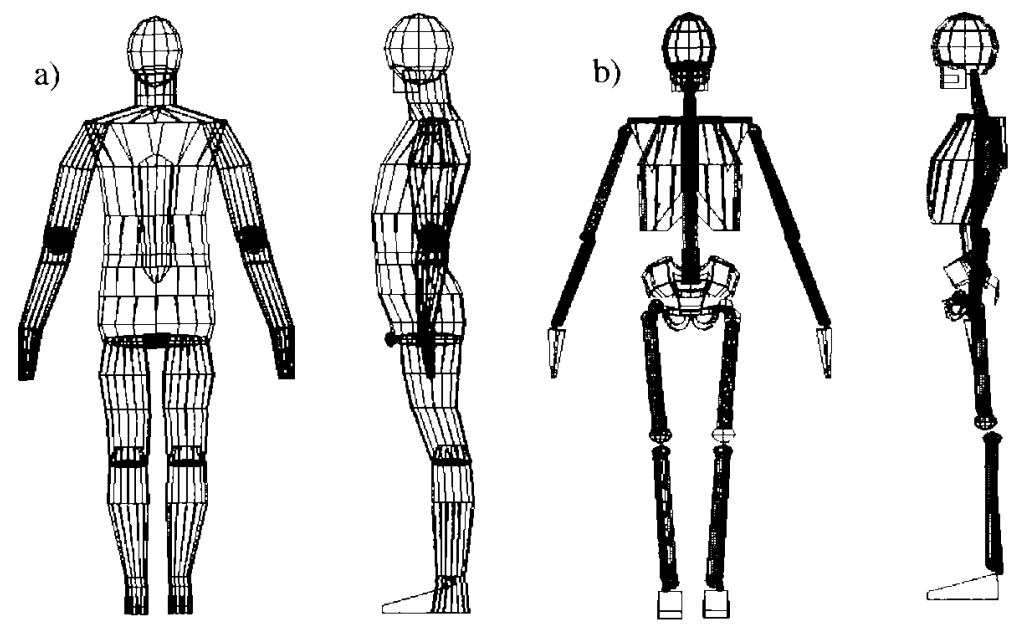

Figure 1 - Representation of the phantom's envelope (a) and its skeleton (b), front and side views.

Représentation de l'enveloppe du fantôme (a) et de son squelette (b), vues face et profil.

is followed throughout its lifetime. This is determined using pseudo-random numbers whose frequency distribution is governed by the various physical interaction laws applicable to the particle in the medium through which it is passing. Every time there is a collision, a statistical weight is assigned to the particle, corresponding to the probability of its emergence after the collision. The life of a particle comes to an end when it meets an "empty" region constituting the geometrical limit of the problem or when its energy drops below the cut-off energy. Biases can also be used to improve the statistics (Bielajew and Rogers, 1988). At each calculation point, the code gives the fluence energy distribution, normalised to one source particle; the total fluence is obtained taking into account the activity of the source and the duration of exposure. The absorbed dose is then obtained by weighting the fluence by a Kerma-fluence conversion factor (Hubbell, 1982) and by roughly assuming that the Kerma represents the dose.

\section{Qualification of the anthropomorphic model}

The aim of the work described here was to qualify the numerical anthropomorphic model created using the combinational geometry principle. First of all, the existing model was studied to improve it and assess the influence of modelling accuracy and morphological parameters on the absorbed dose results. Then, comparisons were made with another anthropomorphic model to assess the influence of the geometric model as a whole. 
TABLE I

Comparison between weights of different organs in the model and ICRP Publication 23 (1975).

Comparaison entre les poids des différents organes dans le modèle et dans la publication 23 de la CIPR (1975).

\begin{tabular}{|ccc|}
\hline $\begin{array}{c}\text { Organs } \\
(\mathrm{g})\end{array}$ & IPSN model & Data ICPR 23 \\
\hline brain & 1500 & 1400 \\
lungs & $1070^{*}$ & $\approx 1100$ \\
heart & $600^{*}$ & 700 \\
stomach & $400^{*}$ & 150 \\
liver & 1800 & $1570-1800$ \\
kidneys & 300 & 310 \\
colon & $720^{* * * *}$ & $725^{* *}$ \\
intestine & $1070^{* * * *}$ & $1040^{* *}$ \\
oesophagus & 40 & 40 \\
bladder & $255^{*}$ & 250 \\
gonads & 38 & 35 \\
\hline
\end{tabular}

* Represents the contain and the container.

** The term colon brings together the ascending one, the transverse one, the descending one and the sigmoid one.

The term intestine represents the small intestine.

\subsection{Improvements made to the anthropomorphic model}

The basic anthropomorphic model (Bottollier-Depois et al., 1998), which was developed for reconstruction purposes, was altered on the basis of anatomical data provided in ICRP Publication 23 (1975). The organs represented in the model are the brain, the lungs, the heart, the stomach, the liver, the kidneys, the colon and the intestines. The data for each (volume, mass etc.) were selected in such a way as to be as representative as possible (Tab. I). They represent a compromise between the exact weight and shape and the constraints associated with representation and space in the numerical model.

Organs such as the oesophagus, the bladder or the testicles were then added to the model (Fig. 2). They were made using data taken from various MIRD (Medical Internal Radiation Dose) publications (Snyder et al., 1978). Unlike the thyroid, these organs, which are important because of their sensitivity to radiation, cannot be simulated by a single point, given their shape or size.

The data published in ICRP Publication 23 (1975) were obtained on the basis of average values recorded for a large group of individuals. The characteristics of certain organs are given with a high degree of uncertainty, corresponding to the considerable variations in these parameters. Thus, a difference in the mass of an 


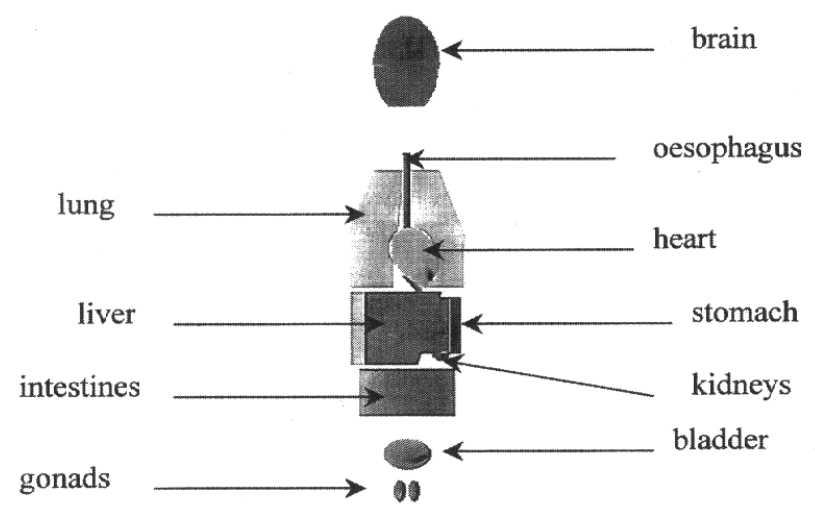

Figure 2 - Representation of organs of the model.

Représentation des organes du modèle.

organ in our model, however great, is not necessarily of any importance and does not imply that our model is unrealistic. Indeed, the stomach for example has a size, a shape and a position which have very important variations. The main factors responsible for these changes are the degree to which the stomach is full, the extent to which the muscles are contracted, the respiration phase and the position of the body. In our case, we modelled the container and the contents in a single solid. This gave a value very close to that of the MIRD model (402 g).

\subsection{The influence of the accuracy of the geometric model}

When a radiological accident occurs, it is useful to have a numerical phantom which can be adapted to better correspond to the morphology of the irradiated individual. However, a lack of morphological information means that approximations are made as regards the model and this leads to uncertainties in the dose distribution calculation. It is not easy to quantify the importance of these approximations for the phantom as a whole since it depends on the configuration of the case being studied. Nevertheless, this effect can be studied and better understood for regions of special interest. Sensitivity tests were carried out for two parts of the human body, the torso and the thigh, which, along with the hand, are the most commonly affected during local irradiation accidents (de Oliveira, 1987). The conclusions were identical in both cases; only the results obtained for the thigh are described here.

The aim of the study was to assess the effect of a change in thigh size on the absorbed dose distribution. The diameter of the thigh model varies within $1 \mathrm{~cm}$; 

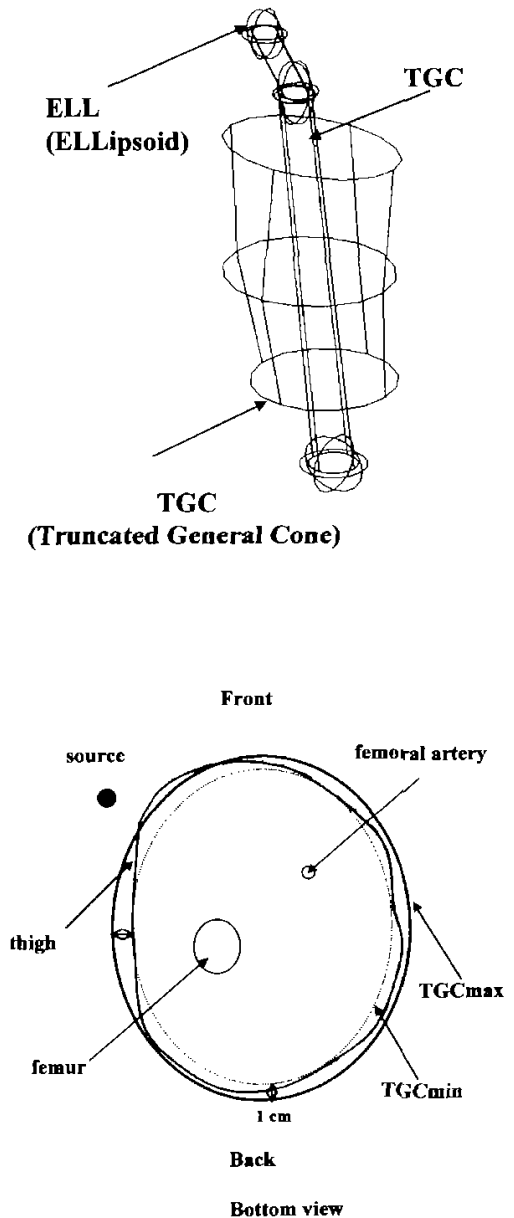

Figure 3-Thigh and femur envelope modelled using the MGED application.

Modélisation de l'enveloppe de la cuisse et du fémur par l'application MGED.
Figure 4 - Cross-section of thigh, modelling of two solids.

Section de la cuisse, modélisation de deux solides.

this value corresponds to the maximum error which can be made when the model is adjusted to the real thigh. A ${ }^{60} \mathrm{Co}$ source was placed at the front of the thigh, simulating a source in the front pocket of a pair of trousers. The thigh and femur used are shown in Figure 3. Using pictures of the patient obtained by means of magnetic resonance imagery techniques, the various parts of the thigh model and the femur position were adjusted. Two sizes of solids (Truncated General Cone) were tested to simulate the thigh. One of the solids completely surrounded the thigh (TGCmax) while the other (TGCmin) was smaller (Fig. 4). They could be considered as representing the highest amount of uncertainty obtained when a part 

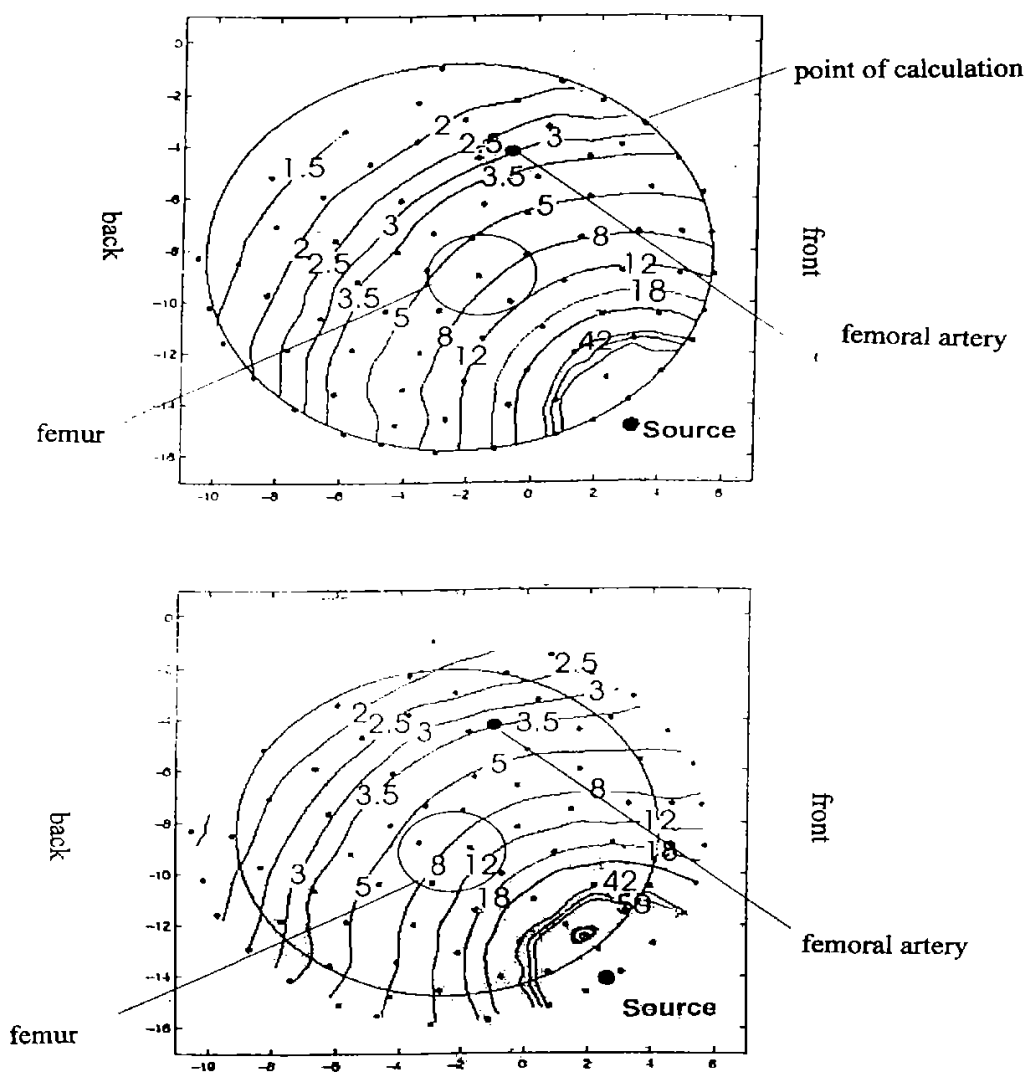

Top view

Figure 5 - Absorbed doses at different points for two sizes of thigh.

Doses absorbées à différents points pour deux tailles de cuisse.

of the body is modelled using this technique (measurement uncertainty of the patient's thigh, model approximation). The two solids differed in size by around $1 \mathrm{~cm}$ along each axis. In both cases, the distance between the source and the skin was kept constant. The points where the doses were of the greatest interest, given the position of the source, were the femoral artery and the femur.

The results show that a decrease of $1 \mathrm{~cm}$ along the axes of the thigh leads to an increase of $15 \%$ in the absorbed dose to the femoral artery and around $30 \%$ to the femur (Fig. 5). These results depend on the morphology of each individual and the 
position of the source, but show the importance of having an accurate model. Since this accuracy cannot be quantified for each patient, special attention should be paid to the early stages of the reconstruction work.

\subsection{The influence of the height and mass parameters of the model on dose calculation}

A series of six models was created so that dose variation as a function of height and mass could be studied. The first of the six models corresponded to the standard anthropomorphic model characterised using the data found in the MIRD publications and ICRP Publication $23(1975)$. It was $170 \mathrm{~cm}$ tall and weighed $70 \mathrm{~kg}$. Two other heights, $155 \mathrm{~cm}$ and $185 \mathrm{~cm}$, were selected. These covered a representative sample of the population and were sufficiently different for the effect of height on dose value to be observed. Two mass values were chosen for each height on the basis of the body mass indexes [mass $(\mathrm{kg}) /$ height $(\mathrm{m})]^{2}$ (Clairand, 1999). These indexes are $23.5 \mathrm{~kg} \mathrm{~m}^{-2}$ and $27 \mathrm{~kg} \mathrm{~m}^{-2}$ respectively and correspond to a person of average build and someone of stout build. Thus, the characteristics of the six models were as follows: $170 \mathrm{~cm}-70 \mathrm{~kg}, 170 \mathrm{~cm}-78.2 \mathrm{~kg}$, $185 \mathrm{~cm}-80.3 \mathrm{~kg}, 185 \mathrm{~cm}-92.4 \mathrm{~kg}, 155 \mathrm{~cm}-56.5 \mathrm{~kg}, 155 \mathrm{~cm}-64.9 \mathrm{~kg}$. They were referred to as models $a, b, c, d, e$ and $\mathrm{f}$ respectively.

The study of the effect of mass and height was carried out using two types of source, ${ }^{192}$ Ir and ${ }^{60} \mathrm{Co}$, placed alongside the left thigh. Calculation points to observe dose variations were placed at different points on the model: the thyroid, the top and base of the spine, the right and left lungs, the stomach, the intestines and the gonads. An average whole body dose was also calculated for each model.

The results, shown in Figure 6, are those obtained from calculations made using the ${ }^{192}$ Ir source. The dose at each point was normalised to 1 for Model a. The first thing we noticed was that height had more effect on the absorbed dose than mass at all points except the gonads. At the various points studied, the absorbed dose decreased as the height increased. The absorbed dose dropped by 12 to $60 \%$ when the height varied between $155 \mathrm{~cm}$ and $170 \mathrm{~cm}$ and by 12 to $55 \%$ (reaching as much as $110 \%$ at the top of the spine) when the height varied between $170 \mathrm{~cm}$ and $185 \mathrm{~cm}$ for a given build. The absorbed dose varied very little (by around 5\%) when the mass varied for a given height. This shows that mass has very little effect on absorbed dose whereas height has a considerable effect. As far as the gonads were concerned, mass and height had an identical effect on the absorbed dose. Indeed, unlike the other calculation points, the gonads were located close to the 

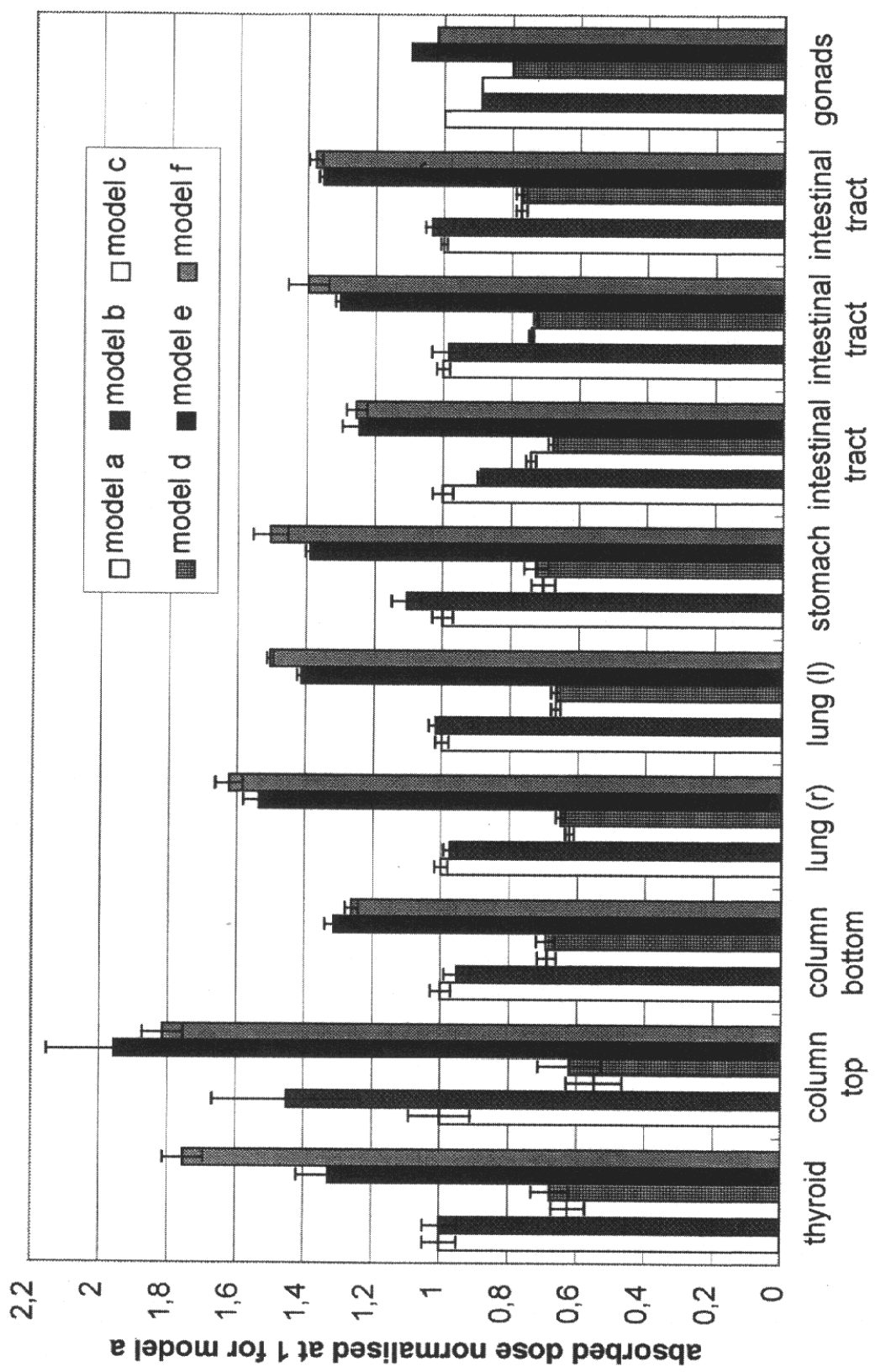


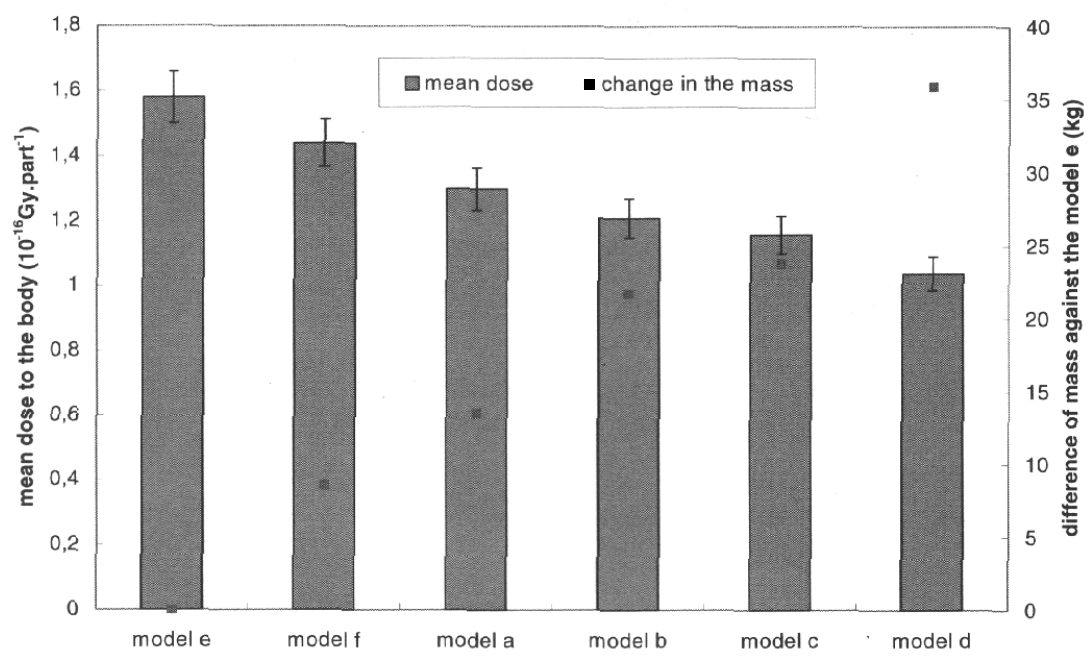

Figure 7 - Variation in average absorbed dose as a function of the weight and height of the model.

Variation de la dose absorbée moyenne en fonction du poids et de la hauteur du modèle.

source and most of the absorbed dose at this point was due to direct radiation. The dose is therefore directly connected to the thickness of the material penetrated and this varies when height and mass are modified. It should also be noted that a variation in mass leads to considerable variation in the absorbed dose to the thyroid in models $e$ and $f$ and to the top of the spine in models $a$ and $b$.

The influence of the two parameters studied is different as regards the whole body average dose (Fig. 7). The average dose results show differences, depending on the models selected but regardless of the source $\left({ }^{192} \mathrm{Ir}\right.$ or $\left.{ }^{60} \mathrm{Co}\right)$. These differences are identical when mass or height is changed. The average dose decreases when one or both of these parameters are increased. Indeed, the average dose is directly related to the volume in which it is measured and mass and height both have an impact on this volume. Furthermore, dose dispersion between the two extremities of the body is greater as the volume increases.

\subsection{Comparisons with other models}

A supplementary study, which contributed to the validation of the numerical model, compared the results obtained with our model and those obtained with 


\section{A. ROUX et al.}

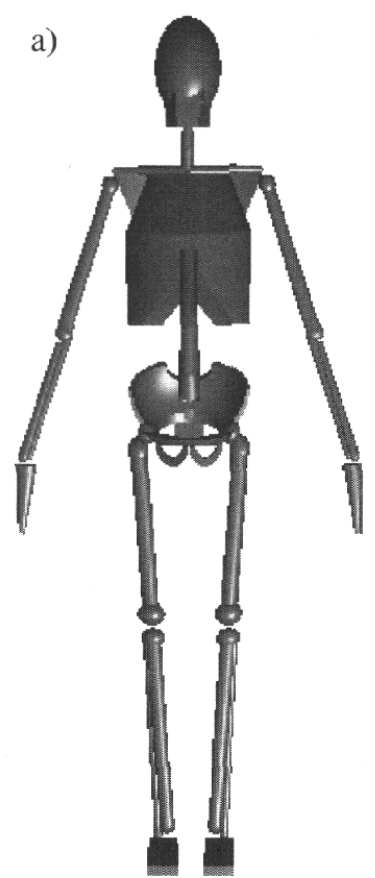

b)

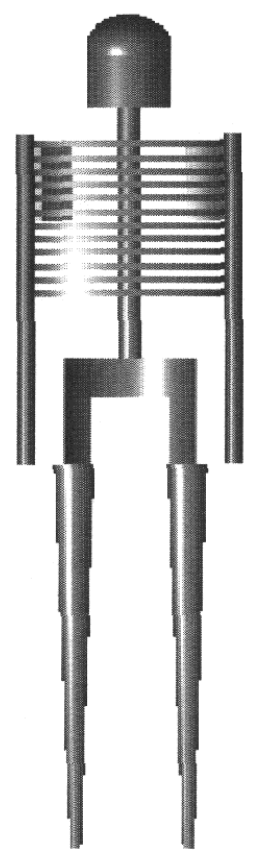

Figure 8 - Representation of the skeleton of our model (a) and that of the MIRD phantom (b) using the MGED application.

Représentation du squelette de notre modèle (a) et de celui du fantôme MIRD (b) utilisant l'application MGED.

another model: the MIRD phantom. This is a standard model commonly used in internal dosimetry. The comparison focused on the regions which were the most sensitive to radiation and on certain organs.

\subsubsection{Comparison of absorbed doses to the skeleton}

To begin with, the comparison focused on the skeleton (Figs. 8a and 8b). Some of its components, such as active bone marrow, are highly sensitive to radioactivity and are spread very evenly throughout the skeleton. Absorbed dose estimates at various points on the skeleton and the average dose (bone and bone marrow) have therefore provided interesting information.

The skeleton of the MIRD phantom was recreated using the MGED application and data taken from the various MIRD publications. This phantom does not 


\section{TABLE II}

Comparison between weights of elements for both skeletons models.

Comparaison entre les poids des éléments des deux modèles de squelettes.

\begin{tabular}{cccc|}
\hline $\begin{array}{c}\text { Skeleton regions } \\
(\mathrm{g})\end{array}$ & $\begin{array}{c}\text { Bone marrow } \\
(\%)\end{array}$ & $\begin{array}{c}\text { IPSN model } \\
(\mathrm{g})\end{array}$ & $\begin{array}{c}\text { MIRD model } \\
(\mathrm{g})\end{array}$ \\
\hline head & 8.3 & $1023^{* *}$ & $1059^{* *}$ \\
scapulas and clavicles & 3.7 & 379 & $283^{*}$ \\
ribs & 18.2 & 955 & 972 \\
spinal column & 30.7 & 1176 & 1289 \\
pelvis & 28.9 & 996 & 848 \\
arms and hands & 2.5 & 1501 & 1363 \\
\hline legs and feets & 7.4 & 4833 & 3920 \\
\hline total & & 10863 & 9734 \\
\hline
\end{tabular}

* Only the scapulas are taken into account in the model.

** Contains the cranium and the jaw.

correspond exactly to the standard MIRD phantom referred to in previous publications. The data relating to the head are different $(-18 \%$ of the mass) as are those of the spine ( $-6 \%$ of the mass), because of geometric representation problems posed by the CAD tool. The doses were calculated using the MORSE code in both cases (the MIRD and our model).

The masses of the various parts of the skeleton are shown in Table II. They have been divided into categories according to their percentage of bone marrow (Cristy, 1981), which must be known if the average dose to the bone marrow is to be obtained from the doses at various points. Generally speaking, the difference in mass between the two models is not very high (around 10\%) over the entire skeleton. However, there are variations at certain points, for example in the "arm" and "leg" regions. Indeed, our model has higher masses in these regions but includes the hands and feet which do not exist in the MIRD model. Likewise, the collarbones are not represented in the MIRD phantom. It should be pointed out that the ribs in our model were simulated using a material comprising bone and soft tissue in identical proportions as regards volume. Only bone mass is shown in Table II.

Irradiation of the skeleton was simulated using a ${ }^{60} \mathrm{Co}$ source placed one metre from the entrance to the model at stomach height. Absorbed dose calculations were made at various points on the skeletons of the two models and an average dose was deduced for each (by weighting the dose at the different points by the mass of the part of the skeleton represented by each of the points).

It can be seen that the results obtained are very similar for a number of calculation points (Fig. 9). On the other hand, there are points at which the absorbed dose is higher for the MIRD model than for ours: the values vary between 20 and 


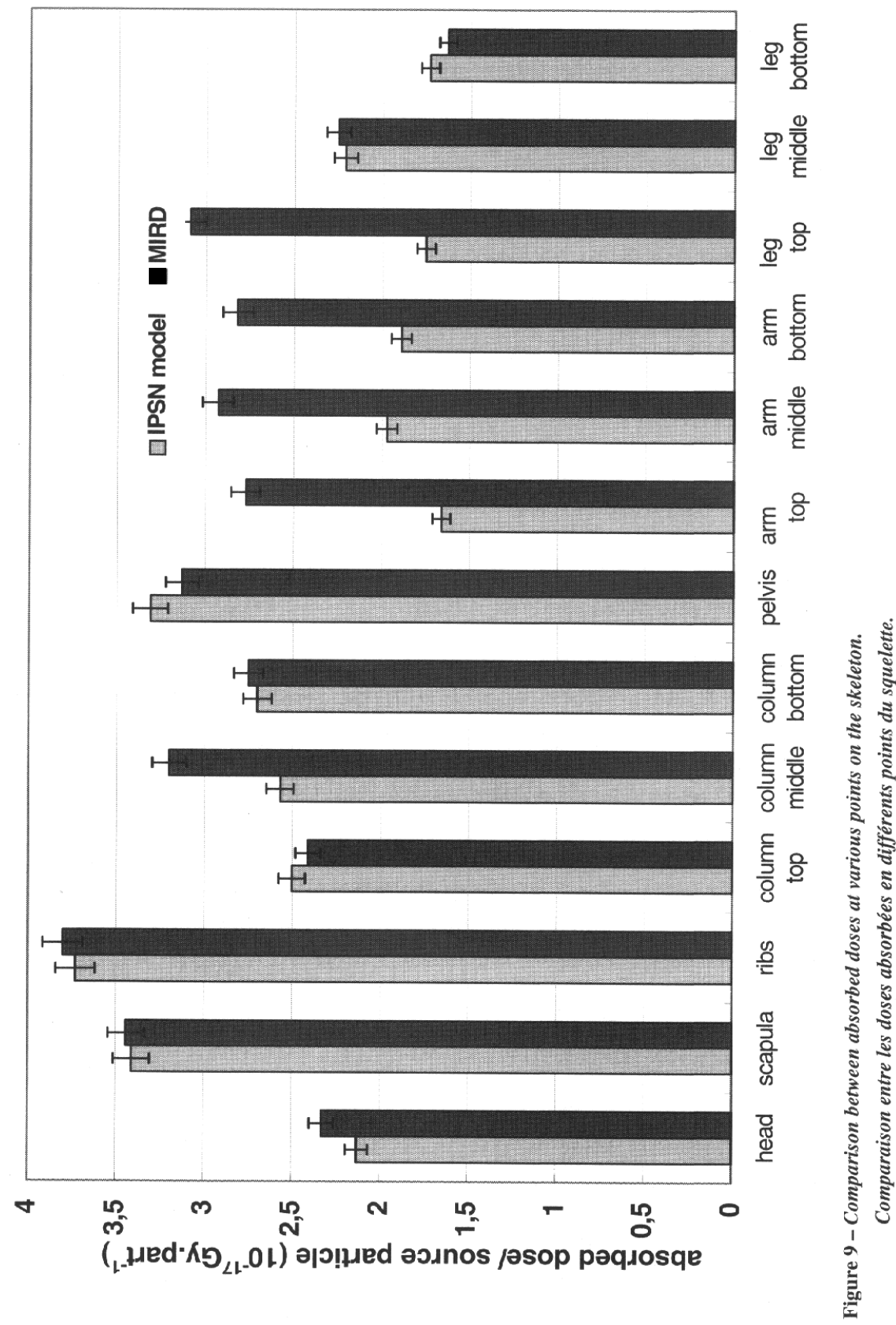


$40 \%$ in the middle of the spine, at the top of the leg and along the entire length of the arm. The differences obtained on the arm are mainly due to the fact that it was in a different position from the rest of the phantom and the source (Figs. 8a and $8 \mathrm{~b}$ ). The difference observed in the middle of the spine can be explained by the fact that the shape of the spine was not the same for the two models. Indeed, whereas in the MIRD phantom the spine was represented by a single vertical cylinder, in our model it was represented by a series of cylinders (Fig. 1), some of which, including that containing the calculation point, are further back than the rest of the skeleton and therefore further from the source. As far as the top of the leg is concerned, the difference is due to a geometric effect, since the pelvis provides more of a screen in our model. As regards the average dose to the skeleton as a whole, the two configurations are more or less identical. The average dose results differ by less than $1 \%$.

\subsubsection{Comparison of absorbed doses to the organs}

In this section, we focus on the average absorbed dose to the organs, calculated using two different phantoms and two different computer codes.

This study was carried out in collaboration with the Moscow Physical Engineering Institute. In the Russian institute, the physics department is studying radiodiagnosis and in this context, implements various dose estimate methods.

The computer code used by the Russian team is the FASTER Monte Carlo code (Lebedev et al., 1989). In this code, each interaction point is treated as a fresh source of direct radiation which contributes to the dose at each detection point. The following interaction point is determined using a Monte Carlo method, Like the MORSE code, it is an energy multi-group code whose effective cross-sections for photons are taken from Hubbell's tables (Hubbell, 1982). The numerical model used by the Russian team is a phantom based on the MIRD phantom (Cristy, 1980). The doses are calculated at various points on the phantom, evenly spread in the volumes of each organ. The statistical uncertainty for the calculation results at various points is less than $3 \%$.

Simulations were carried out by both teams using the same configuration. A ${ }^{60} \mathrm{Co}$ source was placed one metre behind the phantom, facing it at the base of the lungs. The organs studied were the brain, the lungs, the heart, the liver, the stomach and the gonads, along with the skeleton which was available to both teams. These organs were chosen because of their varying degrees of sensitivity to radiation and their positions throughout the body. The results of the average dose comparison for each organ are shown in Figure 10. They show that there is little difference between the two models. Indeed, they differ by less than $7 \%$. In this comparison, 


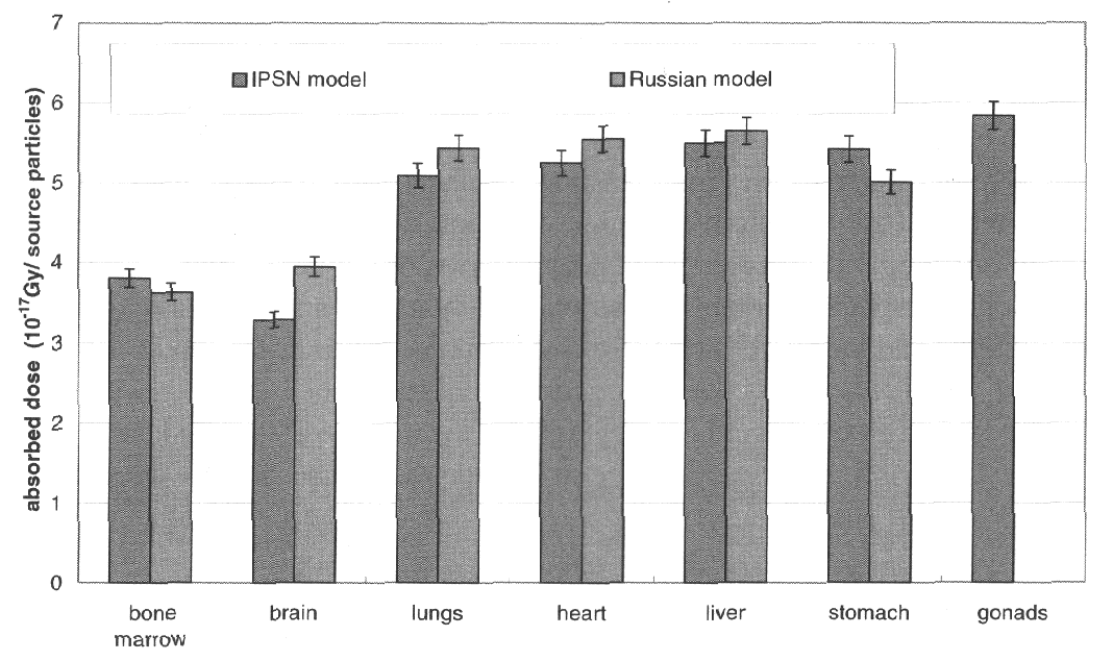

Figure 10 - Comparison between absorbed doses to the organs in two anthropomorphic models.

Comparaison entre les doses absorbées par les organes dans deux modèles anthropomorphiques.

the codes used similar calculation techniques. Furthermore, an earlier study of the MORSE code had shown it to be very similar to other Monte Carlo codes in a scattering medium (Roux et al., 2000). Consequently, the differences in the dose results are mainly due to the differences in the geometric models.

\section{Discussion}

The aim of this study was to qualify and validate the anthropomorphic model in order to obtain a model as close as possible to the one described in ICRP Publication 23 (1975). To this end, a compromise was reached between the height of the model, the exact shape of its constituent parts and the constraints imposed by geometry.

During the study, it became apparent that the accuracy of the model had an important effect on dose calculation. The uncertainty surrounding the dose, which is related to this parameter, increases as the distance between the source and the points studied decreases, since the dose gradient is steeper close to the source.

A sensitivity study of dose as a function of the height and mass of an individual was carried out. When calculating the average dose, the effects of height and mass are comparable since both of these parameters contribute in fine to volume 
variation. On the other hand, the variations in the absorbed dose, observed at different points in the body, show that height has a greater influence. Therefore, if the sources of uncertainty encountered when calculating doses are to be reduced, it will be important to have morphological information on patients, particularly their heights.

Generally speaking, the code developed in the laboratory and the corresponding computer code are satisfactory for reconstructing accidents since they can be put in operation quickly (usually within 48 to 72 hours), regardless of the configuration. The absorbed dose results at different points and the average dose results are accurate enough to be used by medical teams. Furthermore, the physical reconstruction of an accident complements other dosimetric techniques by providing specific information, such as deep dose distributions. The uncertainty corresponding to the dose results is between 15 and $30 \%$ depending on the configuration and includes both statistical and geometric uncertainty. However, the most significant uncertainty is that associated with the geometry of the model. An improvement could be made by developing a more realistic phantom, using scanner images or magnetic resonance images (MRI) then redefining it using a series of small volumes known as voxels. Indeed, experience has shown that MRI data are available for certain local irradiation accidents. In this case, and in order to use all the morphological information to the full, a totally realistic model could be developed. As regards our application, a number of issues still have to be resolved, for example, the development of tools which could be used to describe the contours of organs. Furthermore, as regards accident reconstruction, certain functions should be preserved, such as the possibility of changing the patient's position.

\section{Conclusion}

Once we had validated the calculation tool and assessed the effect of precision on the dose result, we attempted to qualify our numerical anthropomorphic model.

Due to the functions provided by the CAD application, this model is perfectly capable of meeting accident reconstruction requirements. Indeed, based as it is on a model established using standard data, the morphology and position of the model can be changed rapidly. However, it is also a source of uncertainty as regards the dose calculation; this is directly due to the geometric construction mode. This uncertainty depends on the case being studied. In the case of local irradiation, it is important to know the deep dose or the dose at specific points. We therefore tried to obtain a representation of the irradiated part which resembled the individual as much as possible, either by using the data on the patient himself (MRI, scanner, etc.) for positioning organs and bones and determining external contours, or by adjusting the model according to external morphological data (heights, thigh 
measurements, etc.). On the other hand, in the event of global irradiation, where we concentrate on an average dose to the whole body, the accuracy of the geometry is less critical than in the case of local irradiation. External morphological data (height and mass) suffice for adapting the model. This is even more true the further the source is from the individual.

The importance of the geometric model and the corresponding accuracy therefore depend on the type of accident being dealt with. The uncertainty associated with absorbed dose calculations in the case of local irradiation can reach $30 \%$, whereas it would be around $10 \%$ for global irradiation. The absorbed dose accuracy is nonetheless sufficient for it to be interpreted by physicians. Indeed, one symptom cannot be associated with a single dose (Nénot, 1990). It will depend highly on the extent to which the irradiated individual is sensitive to radiation, the start of the dose, the breakdown etc. Moreover, in the case of local irradiation, the deep doses obtained, albeit with a considerable amount of uncertainty, shed light on an in-depth effect which cannot be investigated using other techniques. This information would only be available to the clinic when the symptoms appeared. In all the cases studied, the dose results obtained using our anthropomorphic model comply with those obtained using other models, such as that of the MIRD. The advantage is that the construction tool used makes it possible to preserve the functions which are indispensable for reconstruction, namely changing position and height, for example.

\section{REFERENCES}

Bielajew A.F., Rogers D.W.O. (1988) Variance reduction techniques. In: Monte Carlo transport of electrons and photons (Jenkins T.M., Nelson W.R., Rindi A., Eds.), pp. 407-420, Plenum Press, New York.

Bottollier-Depois J.F., Chau Q., Gaillard-Lecanu E., Medioni R., Roux A. (1998) Techniques of physical dosimetry dedicated to radiological accidents, J. Chim. Phys. 95, 685-690 (in French).

BRL, U.S. Army Ballistic Research Laboratory (1992) The Ballistic Research laboratory CAD Package, Release 4.0, Vol. IV, U.S. Government Printing Office.

Clairand I. (1999) Internal dosimetry for organs. In: Development of news physical models dedicated to internal dosimetry interne par l'utilisation du code de Monte Carlo EGS4, Thesis, pp. 5-24. Université Paul Sabatier, Toulouse.

Cristy M. (1980) Mathematical phantoms representing children of various ages for use in estimates of internal dose. Oak Ridge National Laboratory, Report ORNL-1 159.

Cristy M. (1981) Active bone marrow distribution as a function of age in humans, Phys. Med. Biol. 26 (3), 389-400.

De Oliveira R.A. (1987) Un répertoire des accidents radiologiques 1945-1985. Radioprotection 22, $89-135$.

Emmett M.B. (1985) MORSE-CGA. A Monte Carlo Radiation Transport Code with Array Geometry Capability. Oak Ridge National Laboratory, Report ORNL-6174.

Hubbell J.H. (1982) Photon Mass Attenuation and Energy-absorption coefficients from $1 \mathrm{keV}$ to 20 MeV, Int. J. Appl. Radiat. Isot. 33, 1287. 


\section{NUMERICAL ANTHROPOMORPHIC MODEL}

IAEA (1998) Diagnosis and Treatment of Radiation Injuries, IAEA Safety Reports Series No. 2. IAEA, Vienna.

ICRP Publication 23 (1975) Report of the task group on Reference Man. Pergamon Press, Oxford.

Lebedev L.A., Stavitski R.V., Ermakov I.A. et al. (1989) Organ dose equivalents from X-ray diagnostic examinations. Energoatomizdat, Moscow (in Russian).

Lefaix J.L., Daburon F. (1998) Diagnosis of acute localized irradiation lesions: review of the French experimental experience, Health Phys. 75, 375-384.

Nénot J.C. (1990) Medical and surgical management for localized radiation injuries, Int. J. Radiat. Biol. 57, 783-795.

Roux A., Bottollier-Depois J.F., Gaillard-Lecanu E. (2000) Qualifying numerical tools for reconstructing physical doses in the case of accidental exposure to ionising radiation, Radiat. Prot. Dosim. 87, 243-249.

Snyder W.S., Ford M.R., Warner G.G., Fisher H.L.Jr. (1978) Estimates of absorbed fractions for monoenergetic photon source uniformly distributed in various organs of a heterogeneous phantom. Medical Internal Radiation Dose Committee (MIRD), Pamphlet No. 5, Revised. 